\title{
Synthesis of New Cholesterol and Sugar Anchored Squaraine Dyes: Further Evidence How Electronic Factors Influence Dye Formation
}

\author{
Kuthanapillil Jyothish, Rekha R. Avirah and Danaboyina Ramaiah*
}

Photosciences and Photonics Division

Regional Research Laboratory (CSIR)

Trivandrum 695 019, INDIA

*To whom correspondence should be addressed at:

Photosciences and Photonics Division

Regional Research Laboratory (CSIR)

Trivandrum 695 019, INDIA

Tel: +91 471 2515362; Fax: +91 4712490186 or 914712491712

E-mail: d_ramaiah@ rediffmail.com or rama@csrrltrd.ren.nic.in

\section{Supporting Information}

Synthetic details of the quinaldines, quinaldinium salts, semisquaraines and squaraine

dyes, ${ }^{1} \mathrm{H}$ NMR spectra of the representative semisquaraines and the squaraine dyes, the absorption spectra showing the progress of the reaction and theoretical calculation details. 


\section{Experimental Section}

General techniques. All melting points are uncorrected and were determined on a MelTemp II melting point apparatus. The IR spectra were recorded on a Perkin Elmer Model 882 infrared spectrometer. The electronic absorption spectra were recorded on a Shimadzu UV-3101 or 2401 PC UV-VIS-NIR scanning spectrophotometer. ${ }^{1} \mathrm{H}$ and ${ }^{13} \mathrm{C}$ were measured on a $300 \mathrm{MHz}$ Bruker advanced DPX spectrometer. The fluorescence spectra were recorded on a SPEXFluorolog F112X spectrofluorimeter. All the solvents used were purified and distilled before use. The progress of the reaction was monitored by recording absorption spectrum of the reaction mixture at various time intervals. For this $25 \mu \mathrm{L}$ of the reaction mixture was taken out and diluted to $3 \mathrm{~mL}$ and recorded the absorption spectra.

Starting materials. 4-aminophenol (mp 188-189 $\left.{ }^{\circ} \mathrm{C}\right),{ }^{1}$ 4-iodoaniline (mp 63-64 $\left.{ }^{0} \mathrm{C}\right),{ }^{1}$ 6hydroxy-2-quinaldine (mp 262-264 $\left.{ }^{0} \mathrm{C}\right),{ }^{2}$ 6-iodo-2-quinaldine (mp 108-109 $\left.{ }^{0} \mathrm{C}\right),{ }^{3}$ 6-hydroxy-Nmethyl-2-quinaldinium iodide (2a, mp 238-239 ${ }^{0} \mathrm{C}$ ), ${ }^{2}$ 6-iodo-N-methyl-2-quinaldinium iodide (mp 222-223 ${ }^{0} \mathrm{C}$ ), ${ }^{3}$ 2,3,4,5-tetra-O-benzoyl- $\beta$-D-glucopyranosyl bromide ${ }^{1}$ were prepared by modifying the reported procedures. Cholesteryl chloroformate was purchased from Aldrich and used as such while squaric acid was a gift from Professor Waldemar Adam, University of Würzburg, Germany.

Preparation of 6-tetra-O-benzoyl- $\beta$-D-glucopyranose-2-methylquinoline $\mathbf{1 b}$. To a solution of 6-hydroxyquinaldine (500 mg, $3.14 \mathrm{mmol}$.) in $25 \mathrm{~mL}$ of $1 \mathrm{M} \mathrm{NaOH}$, tetrabutylammonium bromide $(1.01 \mathrm{~g}, \quad 3.14 \mathrm{mmol})$ and 2,3,4,5-tetra-O-benzoyl- $\beta$-Dglucopyranosyl bromide $(2.03 \mathrm{~g}, 3.14 \mathrm{mmol})$ in $25 \mathrm{~mL} \mathrm{CHCl}_{3}$ was added drop wise with vigorous stirring for $3 \mathrm{~h}$. The reaction mixture was diluted with $10 \mathrm{~mL} \mathrm{CHCl}_{3}$, the organic layer was separated and washed with water. Removal of the solvent gave a residue, which was subjected to column chromatography over silica gel. Elution of the column with a mixture of ethyl acetate and petroleum ether (1:9) gave 20\% of the quinaldine derivative $\mathbf{1 b}$, (mp 107-108 $\left.{ }^{0} \mathrm{C}\right)$, IR (KBr) $v_{\max } 2976,1740,1612 \mathrm{~cm}^{-1} ;{ }^{1} \mathrm{H} \mathrm{NMR}\left(300 \mathrm{MHz}, \mathrm{CDCl}_{3}\right) \delta$ 8.03-7.87 (9H, m), 7.59-7.13 (16H, m), 6.07-5.58 (4H, m), 4.78-4.47 (3H, m), $2.67(3 \mathrm{H}, \mathrm{s}) ;{ }^{13} \mathrm{C}$ NMR $(75 \mathrm{MHz}$, $\left.\mathrm{CDCl}_{3}\right) \delta 156.12,155.77,155.34,148.38,145.98,135.17,126.09,123.78,120.15,119.97$, $118.92,118.68,118.14,115.76,113.21,110.38,96.31,71.06,70.91,68.12,60.96,22.86$. 
Preparation of 6- $\beta$-D-glucopyranose-2-quinaldine $\mathbf{1 c}$. A mixture of $\mathbf{1 b}$ in dry methanol and sodium methoxide was stirred at room temperature for $12 \mathrm{~h}$. The reaction mixture was poured into ice water, and extracted with ethyl acetate. Removal of the solvent gave a residue, and the products were separated by column chromatography over silica gel. Elution of the column with a mixture of methanol and ethyl acetate (1: 9) gave $90 \%$ of the quinaldine derivative 1c, mp 161-162 ${ }^{0} \mathrm{C}$, IR (KBr) $v_{\max } 3527,3273,2920,1575 \mathrm{~cm}^{-1}$; ${ }^{1} \mathrm{H}$ NMR (300 MHz, $\left.\mathrm{CDCl}_{3}: \mathrm{MeOH}-\mathrm{d}_{4}\right) \delta 8.11(1 \mathrm{H}, \mathrm{d}, \mathrm{J}=8.4 \mathrm{~Hz}), 7.91(1 \mathrm{H}, \mathrm{d}, \mathrm{J}=9.1 \mathrm{~Hz}), 7.51(1 \mathrm{H}, \mathrm{d}, \mathrm{J}=9.3 \mathrm{~Hz})$, $7.43(1 \mathrm{H}, \mathrm{s}), 7.34(1 \mathrm{H}, \mathrm{d}, \mathrm{J}=8.4 \mathrm{~Hz}), 5.07(1 \mathrm{H}, \mathrm{m}), 3.98(1 \mathrm{H}, \mathrm{m}), 3.81(1 \mathrm{H}, \mathrm{m}), 3.58(3 \mathrm{H}, \mathrm{m})$, $3.34(1 \mathrm{H}, \mathrm{s}), 2.7(3 \mathrm{H}, \mathrm{s}) ;{ }^{13} \mathrm{C} \mathrm{NMR}\left(75 \mathrm{MHz}, \mathrm{CDCl}_{3}: \mathrm{CD}_{3} \mathrm{OD}\right) \delta 158.28,156.35,155.95,144.69$, 137.62, 129.52, 128.63, 123.69, 123.56, 111.69, 102.24, 74.61, 71.19, 62.45, 24.65, 24.25.

Preparation of cholesterol linked 6-hydroxy-2-quinaldine derivative 1d. A mixture of 6hydroxyquinaldine $(100 \mathrm{mg}, 0.63 \mathrm{mmol})$, cholesteryl chloroformate $(450 \mathrm{mg}, 1 \mathrm{mmol})$ and pyridine $(5 \mathrm{~mL})$ were heated at $90{ }^{\circ} \mathrm{C}$ for $12 \mathrm{~h}$. Removal of the solvent gave a residue, which was then subjected to column chromatography over silica gel. Elution of the column with a mixture of ethyl acetate and petroleum ether (1:9) gave 85\% of the quinaldine derivative 1d, mp 101-102 ${ }^{0} \mathrm{C}, \mathrm{IR}(\mathrm{KBr}) v_{\max } 2945,1753,1606 \mathrm{~cm}^{-1} ;{ }^{1} \mathrm{H} \mathrm{NMR}\left(300 \mathrm{MHz}, \mathrm{CDCl}_{3}\right) \delta 8.02(2 \mathrm{H}, \mathrm{m}), 7.61(1 \mathrm{H}$, s), $7.52(1 \mathrm{H}, \mathrm{d}, \mathrm{J}=9.1 \mathrm{~Hz}), 7.43(1 \mathrm{H}, \mathrm{d}, \mathrm{J}=9.1 \mathrm{~Hz}), 5.34(1 \mathrm{H}, \mathrm{s}), 4.61(1 \mathrm{H}, \mathrm{s}), 2.74(3 \mathrm{H}, \mathrm{s})$, 2.04-0.67 (43H, m); ${ }^{13} \mathrm{C}$ NMR (75 MHz, $\left.\mathrm{CDCl}_{3}\right) \delta 157.78,151.86,147.48,144.18,139.82$, 138.06, 135.44, 128.74, 125.64, 124.32, 122.28, 121.82, 121.63, 120.61, 116.64, 78.16, 70.69, $55.76,55.69,55.15,48.99,41.32,38.71,38.52,35.84,35.20,34.78,30.92,30.84,27.22$, 27.00, 23.27, 22.84, 21.81, 21.55, 20.05, 18.27, 17.72, 10.85 .

Preparation of 6-acetoxy-2-quinaldine 1e. A mixture of 6-hydroxyquinaldine (100 mg, $0.63 \mathrm{mmol}$ ), acetic anhydride $(450 \mathrm{mg}, 1 \mathrm{mmol})$ and sodium acetate $(5 \mathrm{mg})$ were stirred at room temperature for $12 \mathrm{~h}$. The reaction was stopped by adding crushed ice and then extracted with chloroform. Removal of the solvent gave a residue, which was subjected to column chromatography over silica gel. Elution of the column with a mixture of ethyl acetate and petroleum ether (1:9) gave $80 \%$ of the quinaldine derivative 1e, mp $169-170{ }^{\circ} \mathrm{C}$, IR $(\mathrm{KBr}) v_{\max }$ 2978, 1745, $1605 \mathrm{~cm}^{-1}$; ${ }^{1} \mathrm{H}$ NMR (300 MHz, CDCl$) \delta 7.82(1 \mathrm{H}, \mathrm{d}, \mathrm{J}=7.8 \mathrm{~Hz}), 7.64(1 \mathrm{H}, \mathrm{s})$, $7.36(2 \mathrm{H}, \mathrm{m}), 6.79(1 \mathrm{H}, \mathrm{d}, \mathrm{J}=7.7 \mathrm{~Hz}), 3.26(3 \mathrm{H}, \mathrm{s}), 2.36(3 \mathrm{H}, \mathrm{s}) ;{ }^{13} \mathrm{C} \mathrm{NMR}\left(75 \mathrm{MHz}, \mathrm{CDCl}_{3}\right) \delta$ 
$169.50,155.75,149.00,145.85,137.45,129.88,129.29,127.49,120.29,113.59,24.80$, 21.05 .

\section{General procedure for the preparation of the quinaldinium salts $2 \mathrm{~b}-\mathrm{e}$}

A mixture of the corresponding quinaldine $(1 \mathrm{mmol})$ and methyl iodide $(4 \mathrm{mmol})$ was heated in a sealed tube at $100-105{ }^{\circ} \mathrm{C}$ for $8 \mathrm{~h}$ (for synthesizing $\mathbf{2 c}$, acetonitrile is used as a solvent). The precipitate formed was filtered, washed thoroughly with cold diethyl ether and subjected to column chromatography over silica gel. Elution of the column with a mixture of methanol and ethyl acetate (1:4) gave the corresponding quinaldinium salts $\mathbf{2 b - e}$ in quantitative yields.

2b (95\%): mp 163-164 ${ }^{0} \mathrm{C}$; IR (KBr) $v_{\max }$ 2982, 1730, $1604 \mathrm{~cm}^{-1} ;{ }^{1} \mathrm{H}$ NMR $(300$ $\left.\mathrm{MHz}, \mathrm{CDCl}_{3}\right) \delta 8.86(1 \mathrm{H}, \mathrm{d}, \mathrm{J}=8.7 \mathrm{~Hz}), 8.22(1 \mathrm{H}, \mathrm{s}), 8.06-7.85(11 \mathrm{H}, \mathrm{m}), 7.51-7.30(12 \mathrm{H}, \mathrm{m})$, $6.10(1 \mathrm{H}, \mathrm{m}), 5.99(1 \mathrm{H}, \mathrm{m}), 5.90(2 \mathrm{H}, \mathrm{m}), 5.02(1 \mathrm{H}, \mathrm{m}), 4.88(1 \mathrm{H}, \mathrm{m}), 4.42(3 \mathrm{H}, \mathrm{s}), 3.01(3 \mathrm{H}, \mathrm{s})$; ${ }^{13} \mathrm{C}$ NMR $\left(75 \mathrm{MHz}, \mathrm{CDCl}_{3}\right) \delta 166.12,165.77,165.34,158.38,155.98,145.17,136.09,133.78$, $133.52,130.15,129.97,128.92,128.14,125.76,120.38,113.21,98.31,73.06,71.90,69.02$, $62.93,41.55,24.88$.

2c (90\%): mp 177-178 ${ }^{0} \mathrm{C}$; IR (KBr) $v_{\max } 3532,3278,3041 \mathrm{~cm}^{-1} ;{ }^{1} \mathrm{H}$ NMR $(300$ MHz, DMSO-d $\left.{ }_{6}\right) \delta 9.12(1 \mathrm{H}, \mathrm{d}, \mathrm{J}=8.4 \mathrm{~Hz}), 8.61(1 \mathrm{H}, \mathrm{d}, \mathrm{J}=9 \mathrm{~Hz}), 8.48(1 \mathrm{H}, \mathrm{d}, \mathrm{J}=9.3 \mathrm{~Hz}), 8.12$ $(1 \mathrm{H}, \mathrm{s}), 7.86(1 \mathrm{H}, \mathrm{d}, \mathrm{J}=8.4 \mathrm{~Hz}), 6.21(1 \mathrm{H}, \mathrm{m}), 6.11(1 \mathrm{H}, \mathrm{m}), 5.94(2 \mathrm{H}, \mathrm{m}), 5.32(1 \mathrm{H}, \mathrm{m}), 4.96$ $(1 \mathrm{H}, \mathrm{m}), 4.74(1 \mathrm{H}, \mathrm{m}), 4.31(3 \mathrm{H}, \mathrm{s}), 3.10(3 \mathrm{H}, \mathrm{s}) ;{ }^{13} \mathrm{C}$ NMR $\left(75 \mathrm{MHz}, \mathrm{DMSO}-\mathrm{d}_{6}\right) \delta 160.97$, 156.78, 146.90, 137.35, 134.09, 128.12, 127.32, 117.56, 99.73, 77.02, 76.42, 73.62, 70.70, 62.12, 40.02, 22.58 .

2d (95\%): $\operatorname{mp} 216-217{ }^{0} \mathrm{C}$; IR (KBr) $v_{\max } 1762,1612 \mathrm{~cm}^{-1} ;{ }^{1} \mathrm{H}$ NMR $(300 \mathrm{MHz}$, $\left.\mathrm{CDCl}_{3}: \mathrm{MeOH}-\mathrm{d}_{4}\right) \delta 8.92(1 \mathrm{H}, \mathrm{d}, \mathrm{J}=8.4 \mathrm{~Hz}), 8.57(1 \mathrm{H}, \mathrm{d}, \mathrm{J}=9.9 \mathrm{~Hz}), 8.17(1 \mathrm{H}, \mathrm{s}), 8.05(1 \mathrm{H}$, m), $7.47(1 \mathrm{H}, \mathrm{s}), 5.45(1 \mathrm{H}, \mathrm{s}), 4.60(3 \mathrm{H}, \mathrm{s}), 4.59(1 \mathrm{H}, \mathrm{s}), 3.35(3 \mathrm{H}, \mathrm{s}), 2.05-0.71(43 \mathrm{H}, \mathrm{m}) ;{ }^{13} \mathrm{C}$ NMR $\left(75 \mathrm{MHz}, \mathrm{CDCl}_{3}\right) \delta 160.33,151.84,150.75,145.56,138.68,130.02,129.20,126.01$, $123.40,120.51,120.22$, 80.08, 56.56, 56.04, 49.91, 49.10, 48.81, 48.53, 48.24, 47.96, $42.17,40.62,39.57,39.34,37.69,36.65,36.40,36.02,35.62,31.73,28.01,27.79,27.42$, $24.07,23.63,22.45,22.21,21.10,20.89,18.98,18.44$.

2e (85\%): mp 187-188 ${ }^{0} \mathrm{C}$; IR (KBr) $v_{\max } \quad 1748,1630 \mathrm{~cm}^{-1}$; ${ }^{1} \mathrm{H}$ NMR $(300 \mathrm{MHz}$, DMSO-D $)_{6} \delta 8.68(1 \mathrm{H}, \mathrm{s}), 8.61(1 \mathrm{H}, \mathrm{d}, \mathrm{J}=8.5 \mathrm{~Hz}), 7.76(1 \mathrm{H}, \mathrm{d}, \mathrm{J}=8.5 \mathrm{~Hz}), 7.28(2 \mathrm{H}, \mathrm{m}), 4.24$ 
(3H, s), $3.66(3 \mathrm{H}, \mathrm{s}), 2,86(3 \mathrm{H}, \mathrm{s}) ;{ }^{13} \mathrm{C}$ NMR $\left(75 \mathrm{MHz}, \mathrm{DMSO}-\mathrm{d}_{6}\right) \delta 159.4,154.4,142.0,131.3$, $129.9,126.8,124.2,122.5,109.9,38.6,22.1,13.6$.

\section{General procedure for the synthesis of the semisquaraines 3a-e}

A mixture of the corresponding quinaldinium salt $(0.03 \mathrm{mmol})$, squaric acid $(0.03 \mathrm{mmol})$ and quinoline $(0.5 \mathrm{~mL})$ were refluxed in a mixture of $n$-butanol and benzene $(6 \mathrm{~mL}$ each, $1: 1)$ with azeotropic distillation of water for $24 \mathrm{~h}$. The solvent was distilled off under vacuum to obtain a residue, which was then subjected to column chromatography over silica gel. Elution of the column with a mixture (1:9) of methanol and chloroform gave the semisquaraines 3a-c in good yields.

For synthesizing $\mathbf{3 d}$, the semisquaraine $\mathbf{3 a}(0.03 \mathrm{mmol})$, cholesteryl chloroformate $(0.03$ mmol) and triethylamine $(1 \mathrm{ml})$ were dissolved in THF $(4 \mathrm{~mL})$ and stirred for $6 \mathrm{~h}$ at $25^{\circ} \mathrm{C}$. The solvent was distilled off under vacuum to obtain a residue, which was then subjected to column chromatography over silica gel. Elution of the column with a mixture (1:9) of methanol and chloroform gave $\mathbf{3 d}$.

For synthesizing $\mathbf{3 e}$, the semisquaraine $\mathbf{3 a}(0.03 \mathrm{mmol})$, acetic anhydride $(0.03 \mathrm{mmol})$ and anhydrous sodium acetate $(1 \mathrm{mg})$ were stirred for $6 \mathrm{~h}$ at $25{ }^{\circ} \mathrm{C}$. The solvent was distilled off under vacuum to obtain a residue, which was then subjected to column chromatography over silica gel. Elution of the column with a mixture (1:9) of methanol and chloroform gave $\mathbf{3 e}$.

3a (95\%): ${ }^{4} \mathrm{mp} 150-152{ }^{\circ} \mathrm{C}$ (mixture mp $150-152{ }^{\circ} \mathrm{C}$ ).

3b (80\%): mp 182-184 ${ }^{0} \mathrm{C}$; IR (KBr) $v_{\max } 3029,2943,1762,1591 \mathrm{~cm}^{-1} ;{ }^{1} \mathrm{H}$ NMR (300 $\left.\mathrm{MHz}, \mathrm{CDCl}_{3}\right) \delta 9.29(1 \mathrm{H}, \mathrm{d}, \mathrm{J}=9.3 \mathrm{~Hz}), 8.93(1 \mathrm{H}, \mathrm{s}), 8.12-7.22(23 \mathrm{H}, \mathrm{m}), 6.11(1 \mathrm{H}, \mathrm{s}), 6.03$ $(2 \mathrm{H}, \mathrm{t}, \mathrm{J}=6.3 \mathrm{~Hz}), 5.82(3 \mathrm{H}, \mathrm{m}), 5.56(1 \mathrm{H}, \mathrm{d}, \mathrm{J}=7.2 \mathrm{~Hz}), 4.73(2 \mathrm{H}, \mathrm{t}, \mathrm{J}=6.3 \mathrm{~Hz}), 3.89(3 \mathrm{H}, \mathrm{s})$, $1.81(2 \mathrm{H}, \mathrm{m}), 1.47(2 \mathrm{H}, \mathrm{m}), 0.96(3 \mathrm{H}, \mathrm{t}, \mathrm{J}=7.2 \mathrm{~Hz})$; FAB-MS: $\mathrm{m} / \mathrm{z}$ Calcd for $\mathrm{C}_{53} \mathrm{H}_{45} \mathrm{NO}_{13}$ : 903.303. Found: 903.298 .

3c $(75 \%)$ : mp 196-198 ${ }^{0} \mathrm{C}$; IR (KBr) $v_{\max } 2996,1732,1610 \mathrm{~cm}^{-1}$; ${ }^{1} \mathrm{H}$ NMR $(300 \mathrm{MHz}$, MeOH-d $\left.{ }_{4}\right) \delta 9.16(1 \mathrm{H}, \mathrm{d}, \mathrm{J}=8.9 \mathrm{~Hz}), 8.49(1 \mathrm{H}, \mathrm{d}, \mathrm{J}=9 \mathrm{~Hz}), 8.41(1 \mathrm{H}, \mathrm{d}, \mathrm{J}=9.3 \mathrm{~Hz}), 8.08(1 \mathrm{H}$, s), $7.78(1 \mathrm{H}, \mathrm{d}, \mathrm{J}=8.4 \mathrm{~Hz}), 6.21(1 \mathrm{H}, \mathrm{m}), 6.11(1 \mathrm{H}, \mathrm{m}), 5.94(3 \mathrm{H}, \mathrm{m}), 5.32(1 \mathrm{H}, \mathrm{m}), 4.96(1 \mathrm{H}$, m), $4.74(1 \mathrm{H}, \mathrm{m}), 4.21-1.02(12 \mathrm{H}, \mathrm{m})$; FAB-MS: $\mathrm{m} / \mathrm{z}$ Calcd for $\mathrm{C}_{25} \mathrm{H}_{29} \mathrm{NO}_{9}$ : 487.199. Found: 487.192.

3d (95\%) : mp 201-203 ${ }^{0} \mathrm{C}$; IR (KBr) $v_{\max } 2945,1762,1539,1232 \mathrm{~cm}^{-1} ;{ }^{1} \mathrm{H}$ NMR (300 $\left.\mathrm{MHz}, \mathrm{CDCl}_{3}\right) \delta 8.57(1 \mathrm{H}, \mathrm{d}, \mathrm{J}=9.6 \mathrm{~Hz}), 7.32-7.26(4 \mathrm{H}, \mathrm{m}), 5.43(1 \mathrm{H}, \mathrm{s}), 5.25(1 \mathrm{H}, \mathrm{s}), 4.84(2 \mathrm{H}$, 
m), $4.61(1 \mathrm{H}, \mathrm{s}), 3.65(3 \mathrm{H}, \mathrm{s}), 2.50(2 \mathrm{H}, \mathrm{d}, \mathrm{J}=6.3 \mathrm{~Hz}), 2.01-0.90(48 \mathrm{H}, \mathrm{m}) ;$ FAB-MS: m/z Calcd for $\mathrm{C}_{47} \mathrm{H}_{63} \mathrm{NO}_{6}$ : 738.006. Found: 738.001.

3e (75\%): mp 180-182 ${ }^{0} \mathrm{C}$; IR (KBr) $v_{\max } \quad 2960,1755,1618 \mathrm{~cm}^{-1} ;{ }^{1} \mathrm{H}$ NMR $(300 \mathrm{MHz}$, $\mathrm{CDCl}_{3}$ : DMSO-d 6 1:1) $8.73(1 \mathrm{H}, \mathrm{d}, \mathrm{J}=9.6 \mathrm{~Hz}), 7.12-6.83(4 \mathrm{H}, \mathrm{m}), 5.39(1 \mathrm{H}, \mathrm{s}), 5.29(3 \mathrm{H}, \mathrm{s})$, $4.17(2 \mathrm{H}, \mathrm{m}), 2.77(3 \mathrm{H}, \mathrm{s}), 1.87-1.05(7 \mathrm{H}, \mathrm{m})$; FAB-MS: m/z Calcd for $\mathrm{C}_{21} \mathrm{H}_{21} \mathrm{NO}_{5}: 367.395$. Found: 367.392 .

\section{General procedure for the synthesis of the symmetrical squaraine dyes $4 \mathrm{~b}-\mathrm{e}$}

A mixture of the corresponding quinaldinium salt $(0.06 \mathrm{mmol})$, squaric acid $(0.03 \mathrm{mmol})$ and quinoline $(0.5 \mathrm{~mL})$ were refluxed in a mixture of $n$-butanol and benzene $(6 \mathrm{~mL}$ each, $1: 1)$ with azeotropic distillation of water for $24 \mathrm{~h}$. The solvent was distilled off under reduced pressure to obtain a residue which was chromatographed over silica gel. Elution of the column with a mixture (1:9) of methanol and chloroform gave the corresponding squaraine dyes $\mathbf{4 b} \mathbf{b} \mathbf{e}$ in good yields.

4b (10\%): $\mathrm{mp} 219-220{ }^{0} \mathrm{C}$, IR (KBr) $v_{\max } 3066,2954,1732,1575,1494 \mathrm{~cm}^{-1} ;{ }^{1} \mathrm{H}$ NMR $\left(300 \mathrm{MHz}, \mathrm{CDCl}_{3}\right) \delta 9.17(2 \mathrm{H}, \mathrm{d}, \mathrm{J}=9.3 \mathrm{~Hz}), 7.98-7.86(13 \mathrm{H}, \mathrm{m}$, aromatic), 7.53-7.02 $(31 \mathrm{H}, \mathrm{m}$, aromatic), 7.44-7.30 (4H, m, aromatic), $6.01(3 \mathrm{H}, \mathrm{m}), 5.79-5.73(7 \mathrm{H}, \mathrm{m}), 5.46(1 \mathrm{H}, \mathrm{s}), 4.67-$ $4.59(5 \mathrm{H}, \mathrm{m}), 4.17(2 \mathrm{H} . \mathrm{s}), 3.62(6 \mathrm{H}, \mathrm{s})$; FAB-MS: m/z Calcd for $\mathrm{C}_{94} \mathrm{H}_{72} \mathrm{~N}_{2} \mathrm{O}_{13}: 1580.488$. Found: 1580.492 .

4d (80\%): $\mathrm{mp} 297-298{ }^{0} \mathrm{C}$, IR (KBr) $v_{\max } 2947,2943,1757,1613,1566 \mathrm{~cm}^{-1} ;{ }^{1} \mathrm{H}$ NMR $\left(300 \mathrm{MHz}, \mathrm{CDCl}_{3}\right) \delta 9.31(2 \mathrm{H}, \mathrm{d}, \mathrm{J}=9.6 \mathrm{~Hz}), 7.44-7.30(8 \mathrm{H}, \mathrm{m}$, aromatic), 5.80 (2H, s, olefinic), $5.43(2 \mathrm{H}, \mathrm{s}), 4.60(2 \mathrm{H}, \mathrm{s}), 3.79(6 \mathrm{H}, \mathrm{s}), 2.50(6 \mathrm{H}, \mathrm{s}) ; 2.04-0.69(83 \mathrm{H}, \mathrm{m}) ;$ FAB-MS: m/z Calcd for $\mathrm{C}_{82} \mathrm{H}_{108} \mathrm{~N}_{2} \mathrm{O}_{8}: 1248.842$. Found: 1248.846 .

4e (85\%): mp 329-330 ${ }^{0} \mathrm{C}$, IR (KBr) $v_{\max } 3017,1749,1620,1573 \mathrm{~cm}^{-1} ;{ }^{1} \mathrm{H}$ NMR (300 MHz, DMSO-d $\left.\mathrm{d}_{6}\right) \delta 8.91(2 \mathrm{H}, \mathrm{s}), 8.25(2 \mathrm{H}, \mathrm{d}, \mathrm{J}=8.5 \mathrm{~Hz}), 8.08(2 \mathrm{H}, \mathrm{d}, \mathrm{J}=7.9 \mathrm{~Hz}), 7.90(2 \mathrm{H}, \mathrm{d}, \mathrm{J}$ $=8.5 \mathrm{~Hz}), 7.58(2 \mathrm{H}, \mathrm{d}, \mathrm{J}=7.9 \mathrm{~Hz}), 5.76(1 \mathrm{H}, \mathrm{s}$, olefinic $), 5.36(1 \mathrm{H}, \mathrm{s}), 4.69(6 \mathrm{H}, \mathrm{s}), 2.50(6 \mathrm{H}, \mathrm{s})$; FAB-MS: $\mathrm{m} / \mathrm{z}$ Calcd for $\mathrm{C}_{30} \mathrm{H}_{24} \mathrm{~N}_{2} \mathrm{O}_{6}$ : 508.166. Found: 508.163.

\section{General procedure for the synthesis of the unsymmetrical squaraine dyes 5a-e}

A mixture of the corresponding semisquaraine $(0.06 \mathrm{mmol}), 6$-iodoquinaldinium salt $(0.06$ $\mathrm{mmol}$ ), and quinoline $(0.5 \mathrm{~mL})$ were refluxed in a mixture of $n$-butanol and benzene $(6 \mathrm{~mL}$ each, 1:1) for $12 \mathrm{~h}$. The solvent was distilled off under reduced pressure to obtain a residue which was 
chromatographed over silica gel. Elution of the column with a mixture (1:9) of methanol and chloroform gave the corresponding squaraine dye.

5a (95\%): ${ }^{4} \mathrm{mp} 317-318{ }^{\circ} \mathrm{C}$ (mixture mp $316-318{ }^{\circ} \mathrm{C}$ ).

5b (76\%): mp 347-348 ${ }^{0} \mathrm{C}$; IR (KBr) $v_{\max } 2962,1730,1614,1572,1554 \mathrm{~cm}^{-1} ;{ }^{1} \mathrm{H}$ NMR $\left(300 \mathrm{MHz}, \mathrm{CDCl}_{3}\right) \delta 9.18(1 \mathrm{H}, \mathrm{d}, \mathrm{J}=9.2 \mathrm{~Hz}), 9.01(1 \mathrm{H}, \mathrm{d}, \mathrm{J}=9.1 \mathrm{~Hz}), 8.06-7.85(14 \mathrm{H}, \mathrm{m}), 7.51-$ $7.30(14 \mathrm{H}, \mathrm{m}), 6.10(2 \mathrm{H}, \mathrm{m}), 5.99-5.90(2 \mathrm{H}, \mathrm{m}), 5.02-4.95(3 \mathrm{H}, \mathrm{m}), 4.88(1 \mathrm{H}, \mathrm{m}), 4.54(1 \mathrm{H}, \mathrm{m})$, $3.92(6 \mathrm{H}, \mathrm{s})$; FAB-MS: $\mathrm{m} / \mathrm{z}$ Calcd for $\mathrm{C}_{60} \mathrm{H}_{45} \mathrm{IN}_{2} \mathrm{O}_{12}: 1112.217$. Found: 1112.210.

5c (70\%): mp 343-344 ${ }^{0} \mathrm{C}$; IR (KBr) $v_{\max } 3512,3388,3253,3070,1746,1618,1554 \mathrm{~cm}^{-1}$. ${ }^{1} \mathrm{H}$ NMR (300 MHz, DMSO-d 6 ) $\delta 9.25(1 \mathrm{H}, \mathrm{d}, \mathrm{J}=9.1 \mathrm{~Hz}), 9.13(1 \mathrm{H}, \mathrm{d}, \mathrm{J}=9.5 \mathrm{~Hz}), 7.98-7.90$ $(3 \mathrm{H}, \mathrm{m}), 7.46-7.4(5 \mathrm{H}, \mathrm{m}), 6.22(1 \mathrm{H}, \mathrm{s}), 6.18(1 \mathrm{H}, \mathrm{s}), 6.10-5.98(3 \mathrm{H}, \mathrm{m}), 5.21-5.12(3 \mathrm{H}, \mathrm{m}), 4.88$ $(1 \mathrm{H}, \mathrm{m}), 3.92(6 \mathrm{H}, \mathrm{s})$; FAB-MS: $\mathrm{m} / \mathrm{z}$ Calcd for $\mathrm{C}_{32} \mathrm{H}_{29} \mathrm{IN}_{2} \mathrm{O}_{8}$ : 696.118. Found: 696.112.

5d (80\%): mp 295-296 ${ }^{0} \mathrm{C}$; IR ( $\left.\mathrm{KBr}\right) v_{\max } 3064,1759,1693,1618,1552 \mathrm{~cm}^{-1} ;{ }^{1} \mathrm{H} \mathrm{NMR}$ $\left(300 \mathrm{MHz}, \mathrm{CDCl}_{3}\right) \delta 9.3(1 \mathrm{H}, \mathrm{d}, \mathrm{J}=9.2 \mathrm{~Hz}), 9.24(1 \mathrm{H}, \mathrm{d}, \mathrm{J}=9.1 \mathrm{~Hz}), 7.76-7.72(3 \mathrm{H}, \mathrm{m}), 7.37-$ $7.29(4 \mathrm{H}, \mathrm{m}), 7.09(1 \mathrm{H}, \mathrm{s}), 5.75(1 \mathrm{H}, \mathrm{s}), 5.45(1 \mathrm{H}, \mathrm{s}), 4.61(1 \mathrm{H}, \mathrm{s}), 3.78(3 \mathrm{H}, \mathrm{s}), 3.70(3 \mathrm{H}, \mathrm{s})$, $3.51(3 \mathrm{H}, \mathrm{s}), 2.52(2 \mathrm{H}, \mathrm{m}), 2.04-0.71(39 \mathrm{H}, \mathrm{m})$; FAB-MS: m/z Calcd for $\mathrm{C}_{54} \mathrm{H}_{63} \mathrm{IN}_{2} \mathrm{O}_{5}: 946.394$. Found: 946.388 .

5e (85\%): mp 341-343 ${ }^{0} \mathrm{C}$; IR (KBr) $v_{\max } 3059,2358,1568,1452 \mathrm{~cm}^{-1}$; ${ }^{1} \mathrm{H}$ NMR $(300$ $\left.\mathrm{MHz}, \mathrm{CDCl}_{3}\right)$ 9.35-9.22 (2H, m), 7.77-7.67 (2H, m), 7.47-7.42 (3H, m), 7.16-6.97 (3H, m), 5.85 $(1 \mathrm{H}, \mathrm{s}), 5.79(1 \mathrm{H}, \mathrm{s}), 3.81(6 \mathrm{H}, \mathrm{s}), 3.71(3 \mathrm{H}, \mathrm{s}) ;$ FAB-MS: m/z Calcd for $\mathrm{C}_{28} \mathrm{H}_{21} \mathrm{IN}_{2} \mathrm{O}_{4}: 576.382$. Found: 576.378 .

\section{References}

1) Vogel's Textbook of Practical Organic Chemistry, $5^{\text {th }}$ ed. Furniss, B. S.; Hannaford, A. J.; Smith, P. W. G.; Tatchell, A. R. Eds.; ELBS and Longman Group Ltd.: London, 1989.

2) Doebner, A.; von Miller, W. Ber. 1884, 17, 708.

3) Jha, B. N.; Banerji, J. C. Dyes and Pigments 1983, 4, 77.

4) Jyothish, K.; Arun, K. T.; Ramaiah, D. Org. Lett. 2004, 6, 3965-3968. 
Table S1. Optimized structure parameters of the quinaldinium salts $\mathbf{2 a}$ and $\mathbf{2 e}$

\begin{tabular}{|c|c|c|c|c|c|c|c|}
\hline & & $2 a$ & & & & $2 e$ & \\
\hline $\mathrm{Co}$ & 1.277542 & 2.039276 & 0.186371 & $\mathrm{CO}$ & -1.712997 & 0.880997 & -0.634995 \\
\hline $\mathrm{CO}$ & 2.248993 & 1.036209 & 0.472855 & $\mathrm{C} 0$ & -1.892990 & -0.253998 & 0.196991 \\
\hline $\mathrm{Co}$ & 1.868805 & -0.267532 & 0.680679 & $\mathrm{C} 0$ & -0.804993 & -0.971985 & 0.636993 \\
\hline $\mathrm{Co}$ & 1.765182 & 3.434952 & -0.028030 & $\mathrm{C} 0$ & 0.515991 & -0.591995 & 0.257996 \\
\hline $\mathrm{Co}$ & -1.277985 & -2.268860 & 0.742874 & $\mathrm{C} 0$ & 0.686996 & 0.546997 & -0.589996 \\
\hline $\mathrm{C} 0$ & -2.244965 & -1.259109 & 0.455536 & $\mathrm{C} 0$ & -0.441986 & 1.278992 & -1.021988 \\
\hline $\mathrm{Co}$ & -1.849609 & 0.037460 & 0.250610 & $\mathrm{C} 0$ & 2.892990 & -0.928986 & 0.315994 \\
\hline $\mathrm{CO}$ & -0.467453 & 0.397232 & 0.321228 & $\mathrm{C} 0$ & 3.078995 & 0.200989 & -0.530991 \\
\hline $\mathrm{CO}$ & 0.493317 & -0.620544 & 0.609467 & $\mathrm{C} 0$ & 2.000992 & 0.927994 & -0.975998 \\
\hline $\mathrm{Co}$ & 0.068680 & -1.951600 & 0.817917 & $\mathrm{C} 0$ & 4.110992 & -1.668000 & 0.762985 \\
\hline $\mathrm{C} 0$ & -1.054764 & 2.723007 & -0.176254 & $\mathrm{C} 0$ & 1.423996 & -2.452988 & 1.564987 \\
\hline No & -0.041931 & 1.719070 & 0.114563 & $\mathrm{C} 0$ & -3.903992 & 1.875992 & -0.429993 \\
\hline $\mathrm{O} 0$ & -1.782532 & -3.521652 & 0.928604 & $\mathrm{C} 0$ & -4.890991 & 2.648987 & -1.225998 \\
\hline $\mathrm{HO}$ & 3.309097 & 1.337173 & 0.522293 & $\mathrm{No}$ & 1.642990 & -1.306992 & 0.696991 \\
\hline $\mathrm{Ho}$ & 2.617813 & -1.049500 & 0.903183 & $\mathrm{O} 0$ & -2.763992 & 1.589996 & -1.179993 \\
\hline $\mathrm{H} 0$ & 2.879105 & 3.470322 & 0.076904 & O 0 & -3.937988 & 1.479996 & 0.728989 \\
\hline $\mathrm{HO}$ & 1.502472 & 3.797958 & -1.055344 & $\mathrm{HO}$ & -2.913986 & -0.548996 & 0.502000 \\
\hline $\mathrm{HO}$ & 1.323776 & 4.134384 & 0.728394 & $\mathrm{HO}$ & -0.977997 & -1.845993 & 1.285995 \\
\hline $\mathrm{HO}$ & -3.309097 & -1.550507 & 0.403885 & $\mathrm{HO}$ & -0.306992 & 2.157990 & -1.673996 \\
\hline $\mathrm{HO}$ & -2.619019 & 0.796143 & 0.030426 & $\mathrm{HO}$ & 4.108994 & 0.474991 & -0.815994 \\
\hline $\mathrm{H} 0$ & 0.822159 & -2.72468 & 1.039246 & $\mathrm{HO}$ & 2.137985 & 1.806992 & -1.631989 \\
\hline $\mathrm{H} 0$ & -0.594788 & 3.735764 & -0.321182 & $\mathrm{HO}$ & 5.026993 & -1.193985 & 0.325989 \\
\hline $\mathrm{H} 0$ & -1.779175 & 2.774902 & 0.682114 & $\mathrm{H} 0$ & 4.078995 & -2.735992 & 0.423996 \\
\hline $\mathrm{Ho}$ & -1.598907 & 2.435532 & -1.117279 & $\mathrm{HO}$ & 4.206985 & -1.643997 & 1.878998 \\
\hline \multirow[t]{2}{*}{$\mathrm{H} \mathrm{O}$} & -1.052734 & -4.134384 & 1.117264 & $\mathrm{HO}$ & 0.890000 & -2.113998 & 2.495987 \\
\hline & & & & $\mathrm{HO}$ & 0.797989 & -3.212997 & 1.022995 \\
\hline
\end{tabular}




\begin{tabular}{|l|l|l|l|l|l|l|l|}
\hline & & & & H 0 & 2.393997 & -2.931000 & 1.862000 \\
\hline & & & & H 0 & -5.092987 & 3.616989 & -0.701996 \\
\hline & & & & H 0 & -4.512985 & 2.853989 & -2.257996 \\
\hline & & & & H 0 & -5.841995 & 2.059998 & -1.285995 \\
\hline
\end{tabular}

Table S2. Optimized structure parameters of the semisquaraines 3a and $\mathbf{3 e}$

\begin{tabular}{|c|c|c|c|c|c|c|c|}
\hline \multicolumn{4}{|c|}{$3 \mathbf{a}$} & \multicolumn{4}{|c|}{$3 e$} \\
\hline $\mathrm{C} 0$ & 6.754990 & 0.086000 & -0.183990 & $\mathrm{C} 0$ & 4.461000 & -1.347992 & 1.506989 \\
\hline $\mathrm{C} 0$ & 6.274994 & -0.235992 & 1.093994 & $\mathrm{C} 0$ & 4.653000 & -1.885986 & 0.224991 \\
\hline $\mathrm{Co}$ & 4.909988 & -0.284988 & 1.343994 & $\mathrm{C} 0$ & 3.589996 & -1.949997 & -0.659988 \\
\hline $\mathrm{C} 0$ & 3.976990 & -0.004990 & 0.326996 & $\mathrm{C} 0$ & 2.306000 & -1.471985 & -0.289993 \\
\hline $\mathrm{CO}$ & 4.463989 & 0.287994 & -0.970993 & $\mathrm{Co}$ & 2.128998 & -0.954987 & 1.025986 \\
\hline $\mathrm{C} 0$ & 5.849000 & 0.336000 & -1.206985 & $\mathrm{C} 0$ & 3.211990 & -0.883987 & 1.910995 \\
\hline $\mathrm{C} 0$ & 3.504990 & 0.507996 & -2.019989 & $\mathrm{C} 0$ & 0.811996 & -0.522995 & 1.425995 \\
\hline $\mathrm{C} 0$ & 2.177994 & 0.363998 & -1.793991 & $\mathrm{CO}$ & -0.235992 & -0.668000 & 0.587997 \\
\hline $\mathrm{C} 0$ & 1.665985 & 0.030000 & -0.485992 & $\mathrm{C} 0$ & -0.081985 & -1.241989 & -0.743988 \\
\hline N 0 & 2.590988 & -0.013992 & 0.556000 & N 0 & 1.227997 & -1.504990 & -1.183990 \\
\hline $\mathrm{C} 0$ & 0.316986 & -0.228989 & -0.252991 & $\mathrm{C} 0$ & -1.165985 & -1.556000 & -1.532990 \\
\hline $\mathrm{C} 0$ & -0.739990 & -0.143997 & -1.186996 & $\mathrm{C} 0$ & -2.520996 & -1.246994 & -1.313995 \\
\hline $\mathrm{CO}$ & -0.991989 & 0.207993 & -2.629990 & $\mathrm{C} 0$ & -3.685989 & -1.867996 & -2.043991 \\
\hline $\mathrm{C} 0$ & -2.481995 & -0.097992 & -2.407990 & $\mathrm{C} 0$ & -4.615997 & -0.941986 & -1.239990 \\
\hline $\mathrm{CO}$ & -2.106995 & -0.409988 & -1.013992 & $\mathrm{C} 0$ & -3.362991 & -0.410995 & -0.600998 \\
\hline O 0 & -3.504990 & -0.087997 & -3.067993 & O 0 & -5.808000 & -0.722992 & -1.148987 \\
\hline O 0 & -0.322998 & 0.578995 & -3.573990 & O 0 & -3.836000 & -2.720993 & -2.896988 \\
\hline O 0 & -2.752991 & -0.795990 & 0.073990 & O 0 & -3.127991 & 0.502000 & 0.365997 \\
\hline $\mathrm{C} 0$ & -4.191986 & -0.997986 & -0.056000 & $\mathrm{C} 0$ & -4.191986 & 1.448990 & 0.589996 \\
\hline $\mathrm{C} 0$ & -4.931000 & 0.019989 & 0.799988 & $\mathrm{C} 0$ & -4.099000 & 2.590988 & -0.403000 \\
\hline $\mathrm{C} 0$ & -6.451996 & 0.181992 & 0.741989 & $\mathrm{C} 0$ & -5.149994 & 3.638992 & -0.108994 \\
\hline $\mathrm{CO}$ & -7.214996 & 0.843994 & 1.582993 & $\mathrm{C} 0$ & -5.067993 & 4.784988 & -1.084991 \\
\hline
\end{tabular}




\begin{tabular}{|c|c|c|c|c|c|c|c|}
\hline O 0 & 8.110992 & 0.119995 & -0.351990 & O 0 & 5.462997 & -1.349991 & 2.475998 \\
\hline $\mathrm{C} 0$ & 2.101990 & -0.063995 & 1.934998 & $\mathrm{C} 0$ & 1.469986 & -1.763992 & -2.578995 \\
\hline $\mathrm{HO}$ & 6.987991 & -0.459000 & 1.880997 & $\mathrm{C} 0$ & 6.720993 & -0.881989 & 2.163986 \\
\hline $\mathrm{HO}$ & 4.582993 & -0.571991 & 2.336000 & $\mathrm{CO}$ & 7.634995 & -0.939987 & 3.338989 \\
\hline $\mathrm{H} 0$ & 6.198990 & 0.566986 & -2.211990 & $\mathrm{O} 0$ & 6.955994 & -0.495987 & 1.020996 \\
\hline $\mathrm{HO}$ & 3.864990 & 0.762985 & -3.012985 & $\mathrm{HO}$ & 5.651993 & -2.238998 & -0.075989 \\
\hline $\mathrm{HO}$ & 1.461990 & 0.490997 & -2.599991 & $\mathrm{HO}$ & 3.761993 & -2.394989 & -1.651993 \\
\hline $\mathrm{HO}$ & 0.010986 & -0.569992 & 0.727997 & $\mathrm{Ho}$ & 3.071000 & -0.477997 & 2.923996 \\
\hline $\mathrm{HO}$ & -4.377991 & -2.021988 & 0.284988 & $\mathrm{HO}$ & 0.693985 & -0.090988 & 2.431000 \\
\hline $\mathrm{HO}$ & -4.470993 & -0.915985 & -1.108994 & $\mathrm{HO}$ & -1.252991 & -0.372986 & 0.883987 \\
\hline $\mathrm{HO}$ & -4.676987 & 1.028000 & 0.446991 & $\mathrm{HO}$ & -1.016998 & -2.140000 & -2.462997 \\
\hline $\mathrm{H} 0$ & -4.575989 & -0.054993 & 1.836990 & $\mathrm{H} 0$ & -3.988998 & 1.798996 & 1.637985 \\
\hline $\mathrm{HO}$ & -6.698990 & -1.196991 & 1.084991 & H 0 & -5.188995 & 0.938995 & 0.536987 \\
\hline $\mathrm{HO}$ & -6.785995 & -0.119995 & -0.302994 & $\mathrm{HO}$ & -4.234985 & 2.192993 & -1.441986 \\
\hline $\mathrm{HO}$ & -7.011993 & 1.864990 & 1.237991 & $\mathrm{HO}$ & -3.074997 & 3.046997 & -0.352997 \\
\hline $\mathrm{H} 0$ & -6.924988 & 0.784988 & 2.637985 & $\mathrm{HO}$ & -5.013992 & 4.022995 & 0.935989 \\
\hline $\mathrm{HO}$ & -8.296997 & 0.679993 & 1.522995 & $\mathrm{HO}$ & -6.168991 & 3.171997 & -0.161987 \\
\hline $\mathrm{HO}$ & 8.308990 & 0.334991 & -1.276993 & $\mathrm{HO}$ & -5.225998 & 4.423996 & -2.129990 \\
\hline $\mathrm{HO}$ & 1.174988 & 0.506989 & 2.002000 & $\mathrm{HO}$ & -4.067993 & 5.278000 & -1.030991 \\
\hline $\mathrm{HO}$ & 2.831985 & 0.403000 & 2.596985 & $\mathrm{HO}$ & -5.850998 & 5.545990 & -0.852997 \\
\hline \multirow[t]{6}{*}{$\mathrm{HO}$} & 1.912994 & -1.091995 & 2.270996 & $\mathrm{HO}$ & 0.698990 & -1.239990 & -3.207993 \\
\hline & & & & $\mathrm{HO}$ & 1.425995 & -2.866989 & -2.786987 \\
\hline & & & & $\mathrm{HO}$ & 2.476990 & -1.368988 & -2.879990 \\
\hline & & & & $\mathrm{HO}$ & 8.138992 & 0.049988 & 3.451996 \\
\hline & & & & $\mathrm{HO}$ & 7.074997 & -1.186996 & 4.272995 \\
\hline & & & & $\mathrm{H} 0$ & 8.407990 & -1.725998 & 3.153000 \\
\hline
\end{tabular}




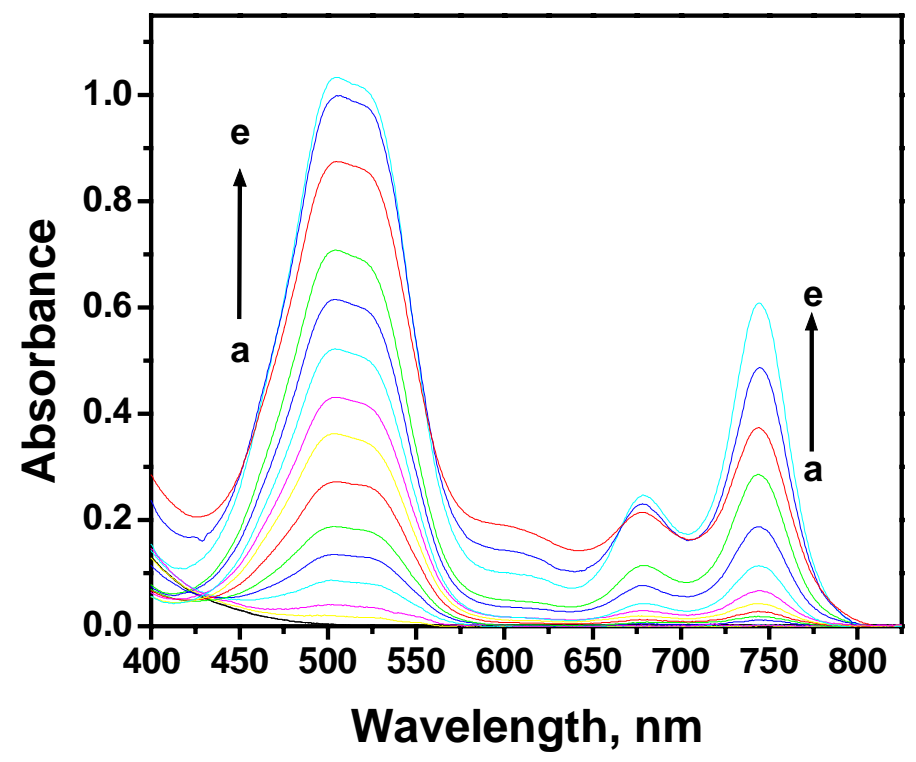

Figure S1. Change in absorption spectra obtained for the reaction between squaric acid and the quinaldinium salt $\mathbf{2 b}$ at various time intervals. Time (a) 0.5, (b) 1, (c) 1.45, (d) 2.45, (e) 4, (f) 5.5, (g) 7.5, (h) 10, (i) 15, (j) 20, (k) 24, (l) $28 \mathrm{~h}$. 


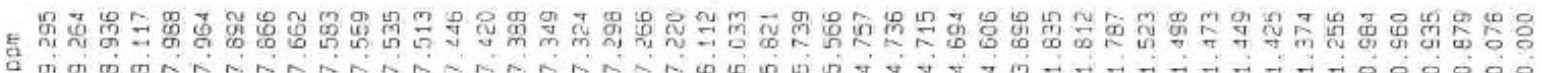

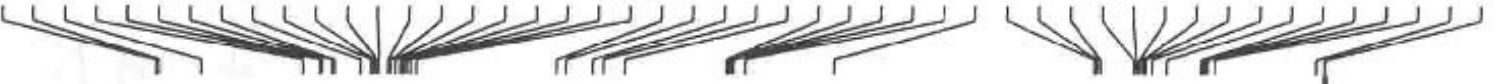

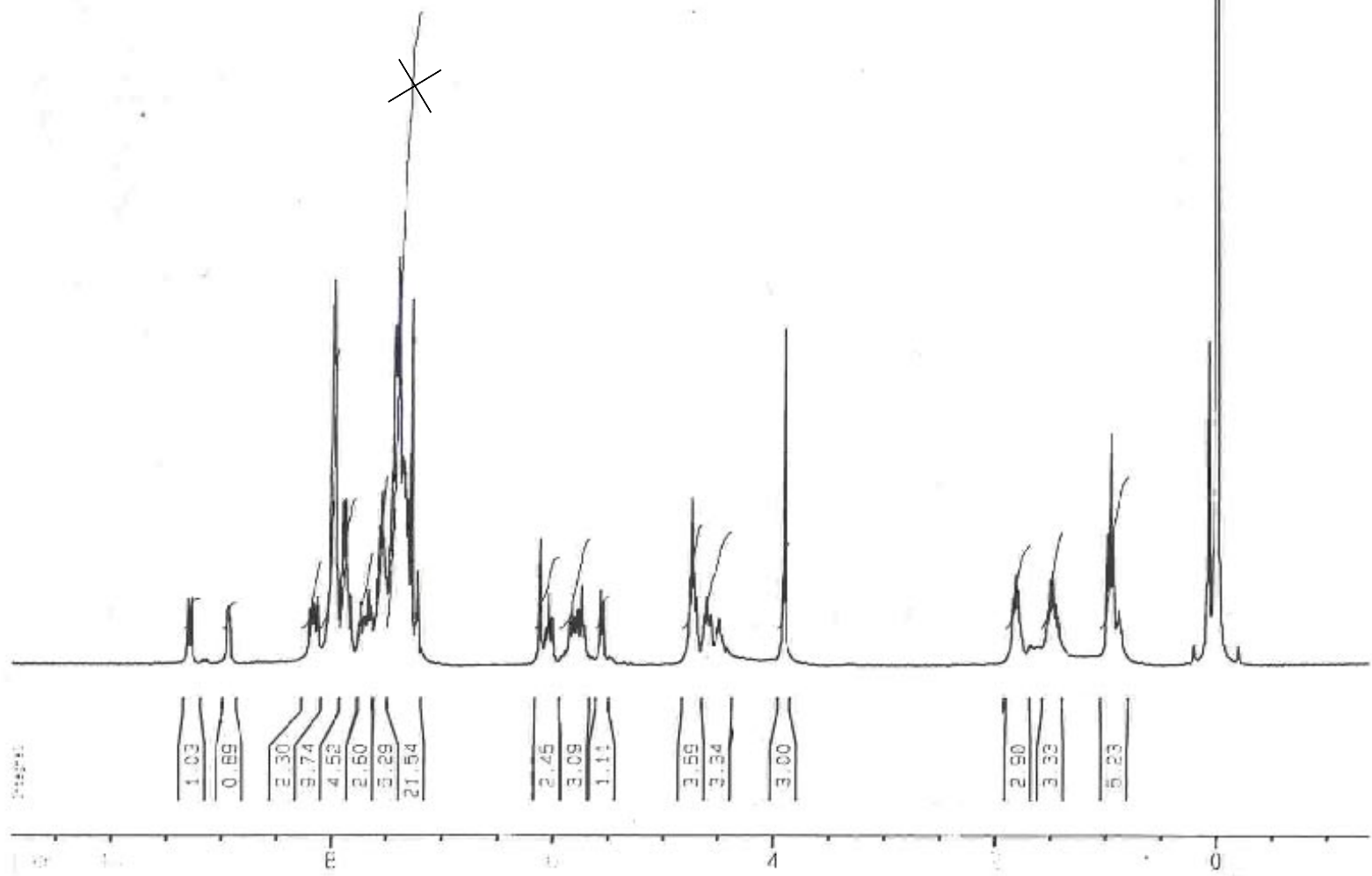

Figure S2. $\quad{ }^{1} \mathrm{H}$ NMR spectra of the semisquaraine derivative $\mathbf{3 b}$ in $\mathrm{CDCl}_{3}$. 


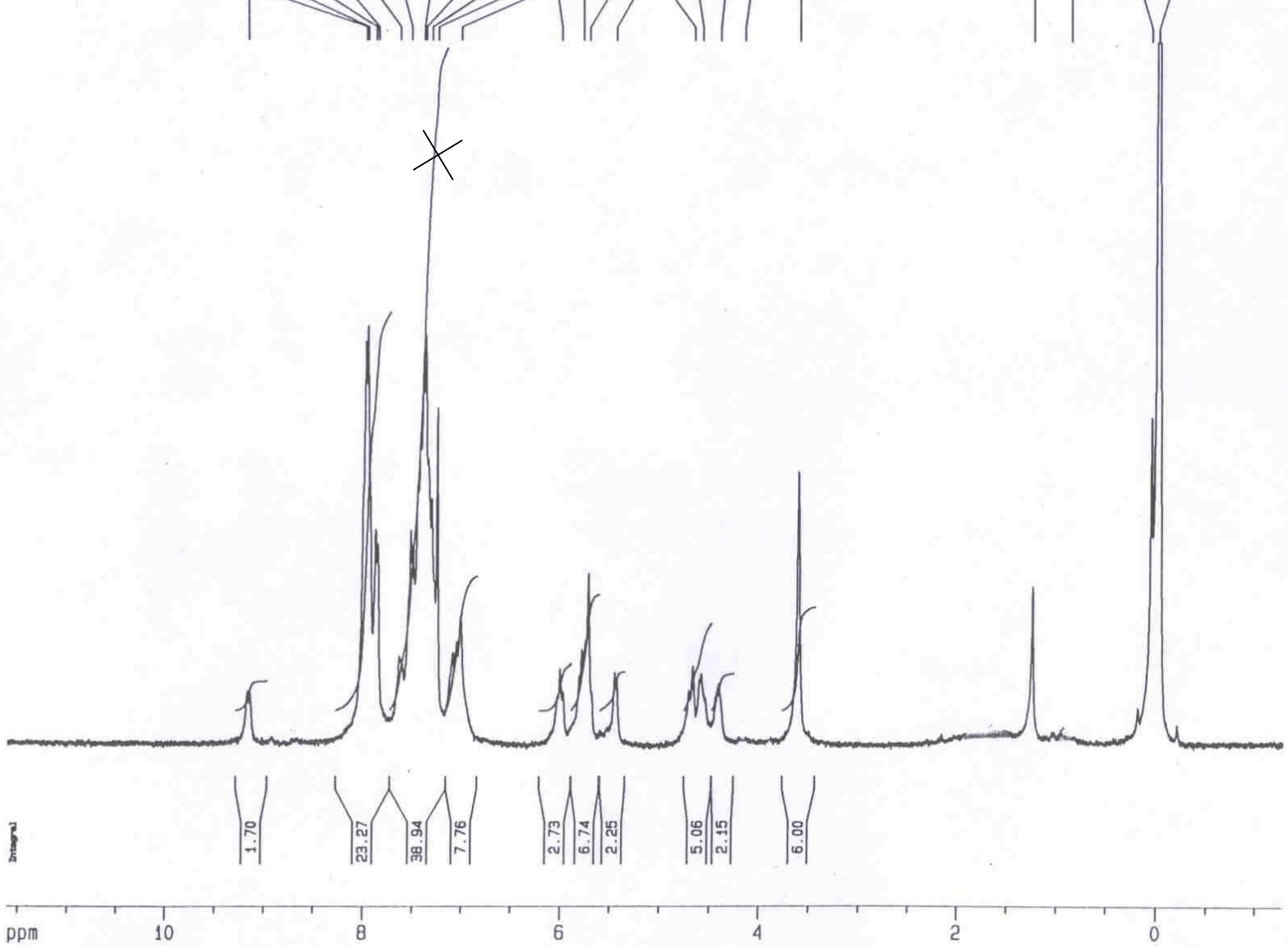

Figure S3. ${ }^{1} \mathrm{H}$ NMR spectrum of the symmetrical squaraine dye $4 \mathbf{b}$ in $\mathrm{CDCl}_{3}$. 


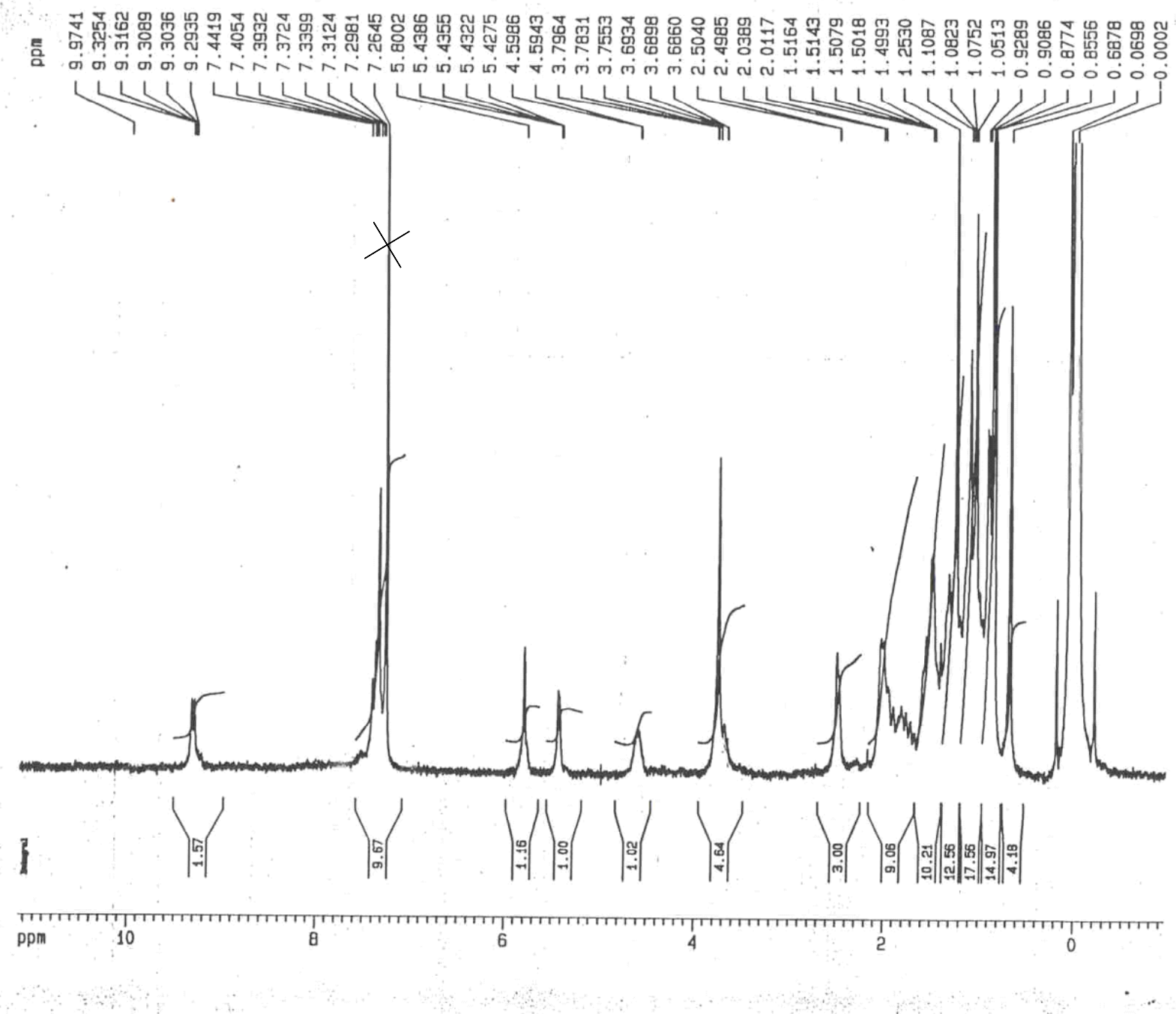

Figure S4. ${ }^{1} \mathrm{H}$ NMR spectrum of the symmetrical squaraine dye $\mathbf{4 d}$ in $\mathrm{CDCl}_{3}$. 


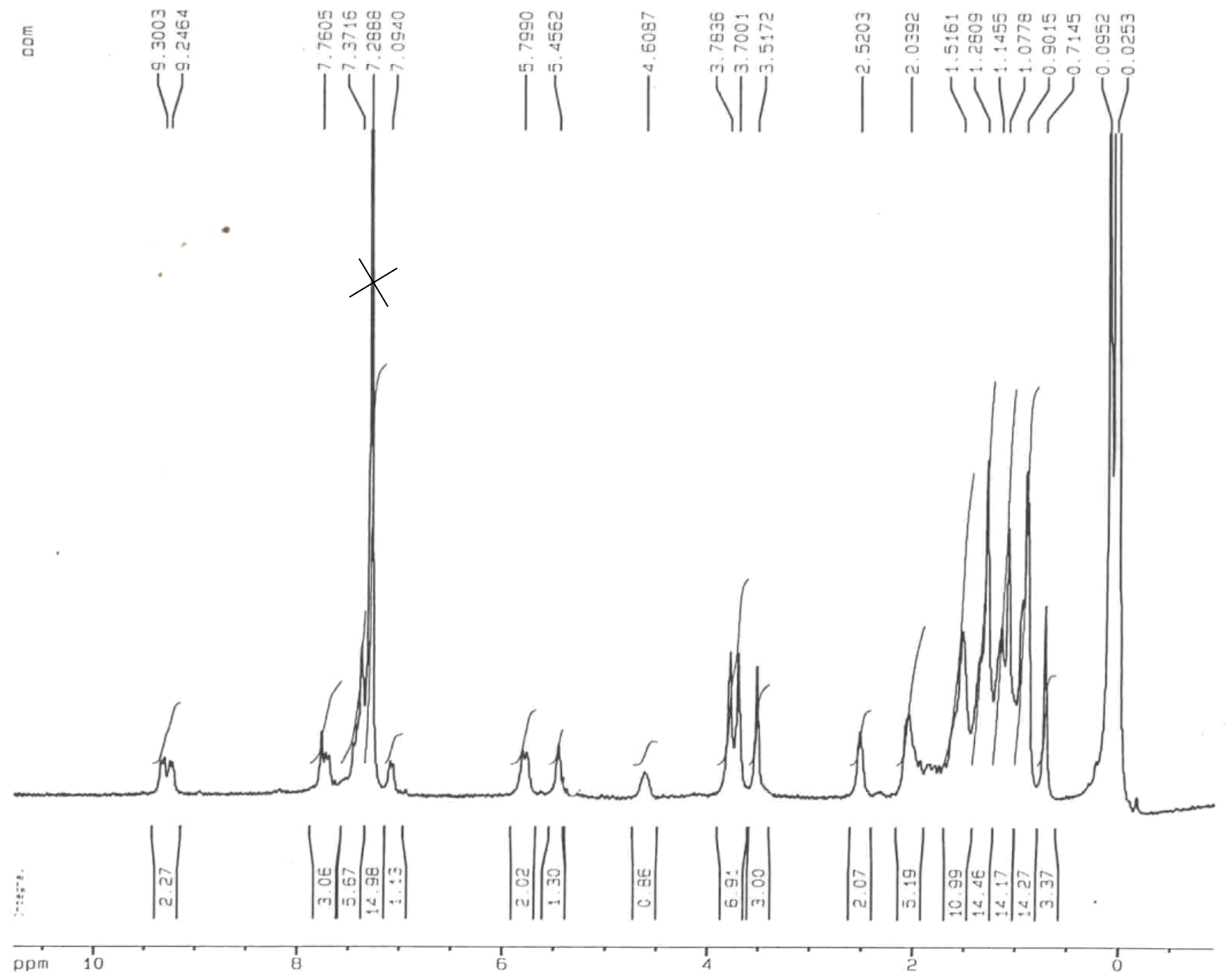

Figure S5. ${ }^{1} \mathrm{H}$ NMR spectrum of the unsymmetrical squaraine dye $\mathbf{5 d}$ in $\mathrm{CDCl}_{3}$. 